\title{
Mechanisms of programmed cell death
}

\begin{abstract}
Objectives
The present review aims to spotlight on the mechanisms and stages of programmed cell death Apoptosis, known as programmed cell death, is a homeostatic mechanism that generally occurs during development and aging in order to keep cells in tissue. It can also act as a protective mechanism, for example, in immune response or if cells are damaged by toxin agents or diseases. In cancer treatment, drugs and irradiation used in chemotherapy leads to DNA damage, which results in triggering apoptosis through the p53 dependent pathway in cancer treatment, drugs and irradiation used in chemotherapy leads to DNA damage, which results in triggering apoptosis through the p53 dependent pathway. Corticosteroids can cause apoptotic death in a number of cells. A number of changes in cell morphology are related to the different stages of apoptosis, which includes nuclear DNA fragmentation, cell shrinkage, chromatin condensation, membrane blebbing, and the formation of apoptotic bodies. There are three pathways for apoptosis, the intrinsic (mitochondrial) and extrinsic (death receptor) are the two major paths that are interlinked and that can effect one another
\end{abstract}

Conclusion: It can be concluded that apoptosis is a homeostatic mechanism that generally occurs during development and aging in order to keep cells in tissue. Drugs and irradiation used in chemotherapy leads to DNA damage, which results in triggering apoptosis through the $\mathrm{p} 53$ dependent pathway. The apoptosis, stages are includes nuclear DNA fragmentation, cell shrinkage, chromatin condensation, membrane blebbing, and the formation of apoptotic bodies. There are three pathways for apoptosis.

Keywords: apoptosis, mechanisms of programmed cell death, stages of apoptosis, pathways for apoptosis
Volume 6 Issue 4 - 2019

\author{
Abdu-Alhameed A Ali Azzwali,' Azab Elsayed \\ Azab $^{2}$ \\ 'Department of Medical Laboratory, Faculty of Medical Technology, \\ Sabratha University, Libya \\ ${ }^{2}$ Department of Physiology, Faculty of Medicine, Sabratha \\ University, Libya
}

Correspondence: : Azab Elsayed Azab, Department of Physiology, Faculty of Medicine, Sabratha University, Libya,

Email azabelsayed@yahoo.com

Received: June 24, 2019 | Published: July 10, 2019

\section{Introduction}

Apoptosis or programmed cell death is characterized by certain morphological and biochemical characteristics. Programmed cell death is a vital process for normal cell turnover, proper development and functioning of the immune system, embryonic development and chemical-induced cell death. Disturbances of apoptosis play a key role in many diseases including neurodegenerative diseases, autoimmune disorders and many types of cancer. ${ }^{1}$ It is a homeostatic mechanism that generally occurs during development and aging in order to keep cells in tissue. ${ }^{2}$ Apoptosis has an important role during an organism's lifecycle. ${ }^{1,3}$ For example, the separation of fingers and toes in a developing human embryo occurs because cells between the digits undergo apoptosis. Apoptosis produces cell fragments called apoptotic bodies that phagocytic cells are able to engulf and quickly remove before the contents of the cell can spill out to surrounding cells and cause damage. ${ }^{1,4}$ It can also act as a protective mechanism, for example, in immune response or if cells are damaged by toxin agents or diseases. ${ }^{5}$ There are many reasons and conditions that can induce apoptosis. For example, in cancer treatment, drugs and irradiation used in chemotherapy leads to DNA damage, which results in triggering apoptosis through the p53 dependent pathway. In addition, some hormones such as corticosteroids can cause apoptotic death in a number of cells. However, it does not have any effect on other cells. ${ }^{2}$ The present review aims to spotlight on the mechanisms of programmed cell death.

\section{The stages of apoptosis}

A number of changes in cell morphology are related to the different stages of apoptosis, which includes nuclear DNA fragmentation, cell shrinkage, chromatin condensation membrane blebbing and the formation of apoptotic bodies (Figure 1). ${ }^{6}$ After the apoptotic bodies are formed, they are rapidly phagocytosed by macrophages that provide non-inflammatory clearance of pre-cancerous and cancerous cells and decrease the damage in tissue that may result from inappropriate immune responses. ${ }^{7}$

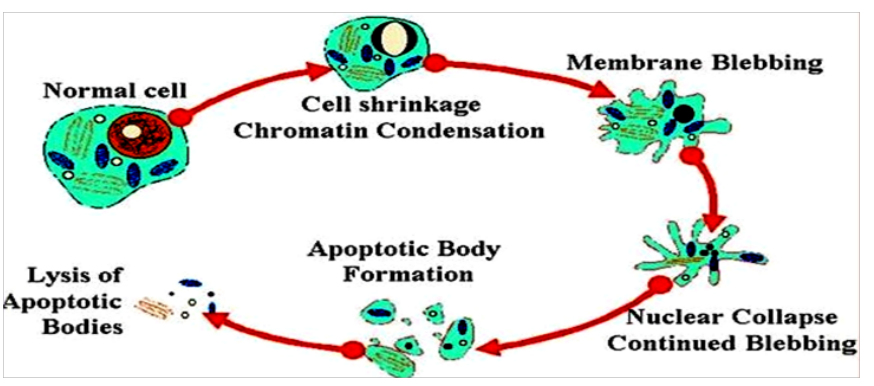

Figure I Morphological changes during apoptosis. ${ }^{7}$

The cell membrane begins to show blebs, decreases in size and chromatin condensation leads to DNA fragmentation and the formation of apoptotic bodies that are phagocytosed.

\section{Mechanisms of apoptosis}

Collecting information and knowledge about apoptotic mechanisms is important and helpful, particularly in targeting the development of drugs. Caspases are essential in this process, which function as both initiators and executioners. ${ }^{8}$ Although there are three pathways for apoptosis, the intrinsic (mitochondrial) and extrinsic (death receptor) are the two major paths that are interlinked and that can effect one another (Figure 2). ${ }^{9}$ 


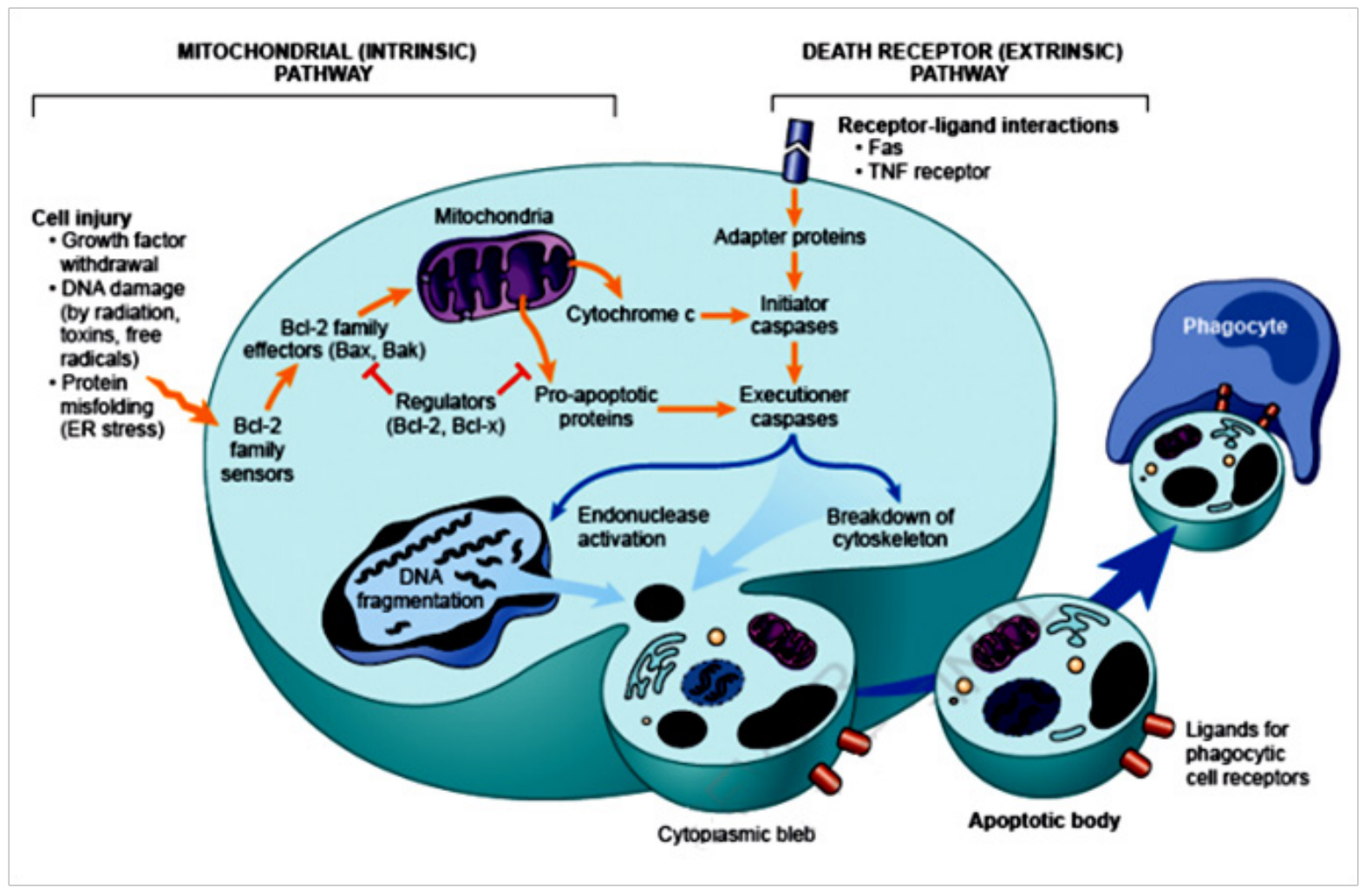

Figure 2 The intrinsic and extrinsic pathways of apoptosis. ${ }^{15}$

\section{The extrinsic death receptor pathway}

As its name suggests, this pathway begins when a death ligand binds to a death receptor. There are a range of different death receptors; however, tumour necrosis factor receptor (TNFR1) andFas(CD95) are the best known among them, with ligands that are called TNF and Fas ligands, respectively. ${ }^{10}$ These receptors have a death domain that require adaptor proteins, such as the TNF receptor associated death domain TRADD and Fas associated death domain FADD. ${ }^{11}$ These proteins bind to the death receptors and giving rise to a ligand-receptor-adaptor protein complex known as the deathinducing signalling complex (DISC). ${ }^{12}$ Pro-caspase 8 is then activated by DISC and the activated enzyme with caspase 8 is able to cleave other downstream or executioner caspases and initiate apoptosis. ${ }^{13}$

\section{The intrinsic mitochondrial pathway}

As its name indicates, this pathway is initiated by internal inducers such as hypoxia and extremely high concentrations of $\mathrm{Ca}^{+2}$ in cytosol. ${ }^{13}$ Regardless of the stimuli, this pathway occurs as a result of increasing the permeability of mitochondria and releasing cytochrome $\mathrm{C}$ into cytosol. ${ }^{14}$ The intrinsic pathway is regulated by two groups of $\mathrm{Bcl}-2$ proteins called anti-apoptotic proteins such as Bcl-2, Bcl-XL, $\mathrm{Bcl}-\mathrm{W}, \mathrm{Bfl}-1$ and $\mathrm{Mcl}-1$.This group regulates apoptosis by blocking the release of cytochrome $\mathrm{C}$ from mitochondria. ${ }^{11}$ However, proapoptotic proteins such as Bax, Bak, Bad, Bcl-Xs, Bid, Bik, Bim and Hrk regulate apoptosis by promoting the release of cytochrome $\mathrm{C}$. The balance between the actions of anti and pro-apoptotic proteins determine the way in which any apoptosis is introduced (Figure 2). ${ }^{15}$

The release of cytc in the intrinsic pathway from the mitochondria leads to formation of the apoptosome and activation of caspase 8 and
9. These activated caspases then activate downstream caspases such as caspase 3, causing cell death. In the extrinsic pathway, specific receptors bind to the ligand forming the DISC complex and activating caspase 8 . The two pathways are connected by the cleaving of the $\mathrm{BH} 3$-only protein BID.

\section{Conclusion}

It can be concluded that apoptosis is a homeostatic mechanism that generally occurs during development and aging in order to keep cells in tissue. Drugs and irradiation used in chemotherapy leads to DNA damage, which results in triggering apoptosis through the p53 dependent pathway. The apoptosis, stages are includes nuclear DNA fragmentation, cell shrinkage, chromatin condensation, membrane blebbing, and the formation of apoptotic bodies. There are three pathways for apoptosis.

\section{Acknowledgments}

None.

\section{Conflicts of interest}

Study has not been ordered, financed and there is no conflict of interest.

\section{References}

1. Kabel AM, Adwas AA, Elkhoely AA, et al. Apoptosis: insights into pathways and role of $\mathrm{p} 53, \mathrm{Bcl}-2$ and sphingosine kinases. Journal of Cancer Research and Treatment. 2016;4:69-722.

2. Elmore S. Apoptosis:a review of programmed cell death. Toxicologic, pathology. Toxicol Pathol. 2007;35(4):495-516. 
3. Davidovich P, Kearney CJ, Martin SJ. Inflammatory outcomes of apoptosis, necrosis and necroptosis. Biol Chem. 2014;395(10):11631171.

4. Chaabane W, User SD, El-Gazzah M, et al. Autophagy, Apoptosis, Mitoptosis and Necrosis: Interdependence Between Those Pathways and Effects on Cancer. Arch Immunol Ther Exp.2013;61:43-58.

5. Norbury CL, Hickson ID. Cellular responses to DNA damage. Annu Rev Pharmacol Toxicol. 2001;41:367-401.

6. Hacker G. The morphology of apoptosis. Cell Tissue Res. 2000;301(1) $5-17$.

7. Hoffmann PR, deCathelineau AM, Ogden CA, et al. Phosphatidylserine (PS) induces PS receptor-mediated macropinocytosis and promotes clearance of apoptotic cells. J Cell Biol. 2001;155(4):649-660.

8. Wong RS. Apoptosis in cancer: from pathogenesis to treatment. $J$ Exp Clin Cancer Res. 2002;30(1):1-14.
9. Igney FH, Krammer PH. Death and anti-death:tumour resistance to apoptosis. Nat Rev cancer. 2002;2(4):277-288.

10. Hengartner MO. Apoptosis corralling the corpses. Cell. 2000;104:325328 .

11. Schneiderer P, Tschopp J. Apoptosis induced by death receptors. PharmActa Helv. 2000;74:281-286.

12. O'brien MA, Kirby R. Apoptosis: a review of pro-apoptotic and antiapoptotic pathways and dysregulation in disease. $J$ Vet Emerg Crit Care. 2008;18(6):572-585.

13. Karp G. Cell and molecular biology. 5th ed, John New Jersey; 2008.

14. Danial NN, Korsmeyer SJ. Cell death: critical control points. Cell. 2004;116(2):205-216.

15. Reed JC. Bcl-2 family proteins: regulators of apoptosis and chemoresistance in haematologic malignancies. Semin Hematol. 1997;34(4 Suppl 5):9-19. 